\title{
Power Optimization of Memoryless Wireless Media Systems with Space-Time Block Codes
}

\author{
Hamid Jafarkhani Homayoun Yousefi'zadeh Mehran Moshfeghi \\ Department of Electrical Engineering and Computer Science \\ University of California, Irvine \\ [hamidj, hyousefi,mmoshfeg] @uci.edu
}

\begin{abstract}
In this research article, we present an analytical solution to the problem of power control in wireless media systems with multiple transmit antennas. We formulate an optimization problem aimed at minimizing total power consumption of wireless media systems subject to a given level of Quality of Service $(\mathrm{QoS})$ and an available bit rate. Our formulation takes into consideration the power consumption related to source coding, channel coding, and transmission of multiple transmit antennas relying on the H.263 source coding standard, Rayleigh fading channels along with the Bernoulli loss model, and space-time block codes respectively.
\end{abstract}

Index Terms - Wireless Media Systems, Power Optimization, Source Coding, Channel Coding, Multiple Antenna Transmission Systems, Space-Time Block Codes, Bernoulli Loss Model, QoS, Rate, Distortion.

\section{INTRODUCTION}

$\mathbf{T}$ HE emergence of new wireless standards is expected to expedite the delivery of the next generation high quality portable multimedia systems. More frequent and longer use of portable multimedia systems is naturally equivalent to higher power consumption of mobile devices. This requires that the power consumption of such devices be kept to a minimum level in order to extend the lifetime of their limited power resources. On the contrary, providing the desired level of quality of service in the presence of the fading effects of multipath wireless channels necessitates higher consumption of power in mobile devices. The theme representing the goal of this research article is, hence, to address the tradeoff between the power consumption and the quality of service in wireless media systems.

In what follows we briefly review some of the specific literature articles in the context of transmitting multimedia content across a wireless backbone. In an early work, Lan et al. [7] solved an energy optimization problem subject to the quality of service when transmitting images across the wireless backbone. However, they did not consider the time varying characteristics of the wireless channel in the analysis of channel coding and transmission. Goel et al. [4] solved another image transmission energy optimization problem subject to distortion and rate constraints. While they appropriately considered hardware specific impacts in their work, their analysis lacked a consideration of channel coding and transmission with respect to the time varying characteristics of the wireless channel. Havinga [5] considered energy efficiency in channel coding techniques for wireless systems without considering the energy of source coding and transmission. Stuhlmuller et al. [11] derived a rate- distortion model for an H.263 compliant coder based on simulation data. Their model could also be used for other codecs that are based on hybrid motion compensation. Appadwedula et al. [2] formulated an energy optimization problem subject to statistical distortion and rate constraints for transmitting images over wireless channels. Considering transmission, source, and channel coding components in the formulation of the problem, the authors deployed a gradient based method to solve the problem. Ji et al. [6] proposed a generic power optimization problem subject to distortion and rate constraints for transmitting video across wireless backbone. The formulation of the latter problem considered the time varying characteristics of memoryless wireless channels relying on joint source channel coding employing Unequal Error Protection (UEP) techniques. The authors solved the problem relying on iterative gradient-based techniques. Lu et al. [8] solved a similar power optimization problem subject to an end-to-end distortion of [11] relying on the H.263 source coding scheme and Reed-Solomon channel coding standard in conjunction with the Gilbert loss model. We point out that none of the literature articles cited above have provided an analytical solution along with an analysis of complexity to their formulated optimization problem. Considering the real-time nature of the problem, we argue that providing an analytical solution to a power optimization problem for wireless systems is attractive as it can potentially offer lower space and time complexity solutions. In addition, none of the articles have considered wireless systems deploying multiple transmit antennas.

The main contribution of this paper is in the following areas. First, the paper proposes the use of multiple antenna transmission systems with space-time block codes in addition to traditional single antenna systems. Second, the paper provides an analytical solution to a formulation of the power optimization problem minimizing the combined power of source coding, channel coding, and transmission while considering rate and distortion constraints.

An outline of the paper follows. In Section II, we provide an analysis of the underlying wireless system consisting of transmitting, channel, and receiving sides. In Section III, we formulate and analytically solve our power optimization problem subject to distortion and rate constraints. In Section IV, we numerically validate our analytical results. Finally, Section V includes a discussion of concluding remarks and future work. 


\section{WiRELESS SYSTEM ANALYSIS}

We start the discussion of this section by reviewing the general model of a communication system utilized to transmit multimedia traffic across a wireless backbone. Fig. 1 includes an illustration of such a system. We note that our model utilizes a multiple transmit antenna system. Generally speaking, optimiz-

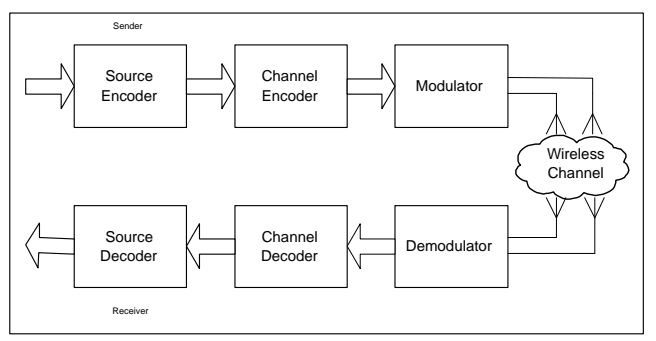

Fig. 1. An illustration of the multiple antenna communication system.

ing power for transmitting and receiving multimedia content to and from a mobile host requires addressing the power consumption tradeoff among different components of the communication system. The latter is of special importance considering the quality of service issues as well as time-varying characteristics of the underlying wireless channel.

\section{A. Source Encoder Analysis}

In this subsection, we provide an analysis for the source coder component of our study based on an H.263 compliant coder. For our source coding analysis, we rely on the experimental results of Stuhlmuller et al. [11]. The experimental distortion model of [11] consists of two components $D_{s}$ and $D_{v}$ respectively imposed by the source encoder and the video decoder. The model relies on an INTRA update scheme forcing a macroblock (MB) to be coded in the INTRA-mode after every $T-1$ macroblocks and resulting in a source encoder distortion of

$$
D_{s}\left(\omega, R_{s}\right)=\frac{\theta(\omega)}{R_{s}-R_{0}(\omega)}+D_{0}(\omega)
$$

where $\omega=\frac{1}{T}$ is the INTRA rate, $R_{s}$ is the encoding bit rate in $K b p s$ and $D_{s}$ is the distortion in terms of the mean square error per source sample. The measurements of [11] also suggest that the distortion-rate parameters $\theta, R_{0}$, and $D_{0}$ depend linearly on the percentage of INTRA coded macro blocks $\omega$ as shown by the following equations.

$$
\begin{aligned}
& \theta=\theta_{P}+\Delta \theta_{P} \omega \\
& R_{0}=R_{0 P}+\Delta R_{0 P} \omega \\
& D_{0}=D_{0 P}+\Delta D_{0 P} \omega
\end{aligned}
$$

The model parameters $\theta_{P}, \Delta \theta_{P}, R_{0 P}, \Delta R_{0 P}, D_{0 P}$, and $\Delta D_{0 P}$ characterize the coding of the input video sequence with the given motion compensated H.263 encoder in baseline mode. It is important to note that the parameters highly depend on the spatial detail and the amount of motion in the sequence.

\section{B. Channel Coder Analysis}

Next, we focus on the analysis of the wireless fading channel and the corresponding channel coder. We utilize the so-called
Rayleigh model with a fading factor $\alpha$ to describe our wireless channel. We note that the output signal of such a channel $S_{o}$ can be related to its input signal $S_{i}$ as

$$
S_{o}=\alpha S_{i}+N
$$

where $N$ indicates the noise signal. Further, we recall that for a multipath slow fading Rayleigh wireless channel, the average received signal to noise ratio is expressed as

$$
\overline{S N R}=\mathcal{E}\left[|\alpha|^{2}\right] \frac{E_{\text {sym }}}{N_{0}}
$$

where $\overline{S N R}$ represents the average received signal to noise ratio of the demodulator of Fig. $1, \mathcal{E}$ denotes the expectation operator, $|\alpha|$ has a Rayleigh distribution, $E_{\text {sym }}$ is the transmission energy per symbol interval, and $N_{0}$ is the onesided spectral density of the white Gaussian noise. Relying on Equation (9.15) of the work of Simon et al. [9] with the choice of $g_{P S K}=\overline{S N R} \sin ^{2}\left(\frac{\pi}{L}\right)$ and $M_{\overline{S N R} 1}(x)=\cdots=$ $M_{\overline{S N R} L}(x)=(1-x \overline{S N R})^{-1}$, one can obtain the symbol error rate of a single transmit $M$ receive antenna system for a slow fading Rayleigh channel utilizing L-PSK modulation scheme as

$$
\begin{aligned}
& e_{\text {sym }}=\frac{L-1}{L}-\frac{1}{\pi} \sqrt{\frac{\vartheta}{1+\vartheta}}\left\{\left(\frac{\pi}{2}+\tan ^{-1} \xi\right) \sum_{j=0}^{M-1}\left(\begin{array}{c}
2 j \\
j
\end{array}\right) \frac{1}{[4(1+\vartheta)]^{j}}\right. \\
& \left.+\sin \left(\tan ^{-1} \xi\right) \sum_{j=1}^{M-1} \sum_{i=1}^{j} \frac{\tau_{i j}}{(1+\vartheta)^{j}}\left[\cos \left(\tan ^{-1} \xi\right)\right]^{2(j-i)+1}\right\}
\end{aligned}
$$

where $\vartheta=\overline{S N R} \sin ^{2}\left(\frac{\pi}{L}\right), \xi=\sqrt{\frac{\vartheta}{1+\vartheta}} \cot \frac{\pi}{L}$ and $\tau_{i j}=$ $\frac{\left(\begin{array}{c}2 j \\ j\end{array}\right)}{\left(\begin{array}{c}(j-i) \\ j-i\end{array}\right) 4^{i}[2(j-i)+1]}$. Noting that the number of bits per symbol $m$ is related to the number of signal points in the constellation $L$ as $m=\log _{2} L$ and for the proper choice of normalization factors $\mathcal{E}\left[|\alpha|^{2}\right]$ and $N_{0}$, the result of Equation (5) for a single receive antenna system where $M=1$ and BPSK modulation scheme where $m=1$ and $L=2$ is expressed as

$$
e_{\text {sym }}=\frac{1}{2}\left\{1-\sqrt{\frac{\overline{S N R}}{1+\overline{S N R}}}\right\}
$$

Similarly, the result for $M=1$ and QPSK modulation scheme where $m=2$ and $L=4$ is expressed as

$$
e_{\text {sym }}=\frac{3}{4}\left\{1-\frac{4}{3 \pi} \sqrt{\frac{\overline{S N R}}{2+\overline{S N R}}}\left[\frac{\pi}{2}+\tan ^{-1} \sqrt{\frac{\overline{S N R}}{2+\overline{S N R}}}\right]\right\}
$$

Further, we note that a single transmit double receive antenna $(1 \times 2)$ system has a $3 \mathrm{~dB}$ SNR advantage over a double transmit single receive antenna $(2 \times 1)$ system utilizing the space-time block codes of [1] and [12]. Hence, the symbol error rate of a $1 \times 2$ system can identify the symbol error rate of such a $2 \times 1$ system by replacing $S N R$ with $\frac{S N R}{2}$ in the symbol error rate of the former. For a double transmit single receive antenna system with BPSK modulation scheme, the symbol error rate is expressed as

$$
e_{\text {sym }}=\frac{1}{2}\left\{1-\sqrt{\frac{\overline{S N R}}{2+\overline{S N R}}}\left[1+\frac{1}{2+\overline{S N R}}\right]\right\}
$$

Similarly for a double transmit single receive antenna system, the symbol error rate of QPSK modulation scheme is expressed as

$$
\begin{aligned}
e_{\text {sym }}= & \frac{3}{4}-\frac{\zeta}{\pi}\left\{\left(\frac{\pi}{2}+\tan ^{-1} \zeta\right)\left(1+\frac{2}{4+\overline{S N R}}\right)\right. \\
& \left.+\sin \left(2 \tan ^{-1} \zeta\right)\left(\frac{1}{4+\overline{S N R}}\right)\right\}
\end{aligned}
$$


where $\zeta=[(\overline{S N R}) /(4+\overline{S N R})]^{1 / 2}$. Having specified the symbol error rate based on the channel characteristics, we propose utilizing a Reed-Solomon channel coder $R S(n, k)$ that converts $k$ information symbols into an $n$-symbol block as the result of appending $(n-k)$ parity symbols. Assuming $R_{s}$ and $R_{c}$ respectively denote source and channel coding bit rates, we note that utilizing such a channel coding scheme introduces a channel code rate $r=\frac{k}{n}=\frac{R_{s}}{R_{s}+R_{c}}$. The scheme also allows for correcting $t_{c}=\left\lfloor\frac{n-k}{2}\right\rfloor$ symbol errors. In order to calculate the error rate of a block utilizing an $R S(n, k)$ coder, we consider the single-state Bernoulli error model representing a binary symmetric channel. In the Bernoulli model, the probability of losing more than $t_{c}$ symbols from $n$ transmitted symbols also known as the residual symbol error rate is given by

$$
\Psi\left(n, t_{c}\right)=\sum_{i=t_{c}+1}^{n}\left(\begin{array}{l}
n \\
i
\end{array}\right) e_{s y m}^{i}\left(1-e_{s y m}\right)^{(n-i)}
$$

where $e_{\text {sym }}$ is the symbol error rate.

\section{Video Decoder Analysis}

Reference [11] also proposes that the video coder distortion caused by transmission errors is expressed as

$$
D_{v}(\omega, \Psi)=\sigma_{u 0}^{2} \Psi \sum_{t=0}^{T-1} \frac{1-\omega t}{1+\lambda t}
$$

where leakage $\lambda$ describes the efficiency of loop filtering to remove the error and $\sigma_{u 0}^{2}$ describes the sensitivity of the video decoder to an increase in error rate. In addition, the residual symbol error rate $\Psi$ which is the probability that a block cannot be recovered at the channel decoder is calculated from Equation (10). The overall distortion $D_{\text {total }}$ at the video decoder is, then, calculated as

$$
D_{\text {total }}=D_{s}+D_{v}
$$

\section{POWER Optimization}

In this section, we focus on power optimization of a mobile device utilized in a wireless media system with space-time block codes. Recalling that the overall power consumed in a mobile device is associated with source coding, channel coding, and transmission, we first introduce individual terms expressing the consumed power of different components. We then proceed with the formulation of the power optimization problem and an analytical solution to it.

\section{A. Power Consumption Models}

The first power consumption component of the underlying wireless system is the source encoder. Reference [8] proposes the following average power consumption model for an H.263 coder

$$
P_{s}\left(\omega, R_{s}\right)=\epsilon_{s}\left(a_{s}-b_{s} \omega+c_{s} R_{s}\right)
$$

where $\epsilon_{s}$ is the weighting factor introduced to allow scaling of the model based on the actual power consumption of a particular implementation, $a_{s}, b_{s}, c_{s}$ are described in terms of the energy consumptions of different source coding components, $\omega$ is again the source coder INTRA rate, and $R_{s}$ again indicates the source coding bit rate. From a practical stand point, the authors of [8] confirm that the power consumption models for encoding the sequences Containership.qcif, Foreman.qcif, MotherDaughter.qcif, News.qcif, and SilentVoice.qcif utilizing an H.263 software encoder along with least square error fitting technique to determine the parameters of Equation (13) are quite accurate.

The second power consumption component of the underlying wireless system is the channel coder. Reference [2] models per bit energy consumption of a Reed-Solomon $R S(n, k)$ encoder as

$$
P_{c}\left(R_{s}, R_{c}\right)=\epsilon_{c} \frac{n R_{s} R_{c}}{m\left(R_{s}+R_{c}\right)}
$$

where $\epsilon_{c}$ is the scaling factor and $m$ is the number of bits per symbol.

Finally, the third power consumption component of the underlying wireless system is the transmitter. The total transmission power is given by

$$
P_{t}\left(R_{s}, R_{c}, E_{s y m}\right)=\epsilon_{t} \frac{E_{s y m}}{m}\left(R_{s}+R_{c}\right)
$$

where $\epsilon_{t}$ is the scaling factor mapping the radiated energy into actual transmission power of a wireless device.

\section{B. Power Optimization Problem}

Having expressed all of the power consumption components as well as the distortion terms, we now formulate our power optimization problem subject to distortion and rate constraints as

$$
\begin{aligned}
\underset{\omega, R_{s}, R_{c}, E_{\text {sym }}}{\min } P_{\text {total }} & =P_{s}+P_{c}+P_{t} \\
\text { Subject To: } & D_{\text {total }}=D_{s}+D_{v} \leq D_{0} \\
R_{\text {total }} & =R_{s}+R_{c} \leq R_{0}
\end{aligned}
$$

We observe that for a single/multiple transmit antenna wireless system utilizing L-PSK modulation scheme, the objective function and inequality constraints of the above optimization problem can be expressed in terms of optimization variables $\omega$, $R_{s}, R_{c}$, and $E_{s y m}$ as well as some constants. The derivation is based on equations (13), (14), (15) along with (1), (2), (4), (5), (10), and (11).

Next, we provide an analytical solution to the optimization problem formulated by Equation (16), and constraint set (17) and (18). Relying on the Lagrangian theory [3], we convert the problem to an optimization problem without constraints. We define the Lagrangian function of Equation (16) as

$$
\begin{aligned}
& L G_{P}=P_{s}+P_{c}+P_{t}+ \\
& \quad \mu_{1}\left(D_{s}+D_{v}-D_{0}\right)+\mu_{2}\left(R_{s}+R_{c}-R_{0}\right)
\end{aligned}
$$

where the parameters $\mu_{1}$ and $\mu_{2}$ are the Lagrange multipliers in the Lagrangian Equation (19). The unconstrained minimization problem for $\Omega=\left\{\omega, R_{s}, R_{c}, E_{s y m}\right\}$ is defined as

$$
\begin{aligned}
& \min _{\Omega} L G_{P}=\min _{\Omega}\left\{P_{s}+P_{c}+P_{t}+\right. \\
& \left.\mu_{1}\left(D_{s}+D_{v}-D_{0}\right)+\mu_{2}\left(R_{s}+R_{c}-R_{0}\right)\right\}
\end{aligned}
$$




\section{Conditions of Optimality: Constraint Qualifications}

We now investigate the existence of necessary and sufficient optimality conditions also known as constraint qualifications. For our unconstrained minimization problem

$$
\min _{\omega, R_{s}, R_{c}, E_{s y m}} L G_{P}
$$

the constraint qualifications are expressed in terms of Lagrange multiplier theory revolving around conditions under which Lagrange multiplier vectors satisfying the following conditions are guaranteed to exist for a local minimum $\Omega^{*}=$ $\left\{\omega^{*}, R_{s}^{*}, R_{c}^{*}, E_{s y m}^{*}\right\}$ satisfying

$$
\nabla L G_{P}\left(\Omega^{*}\right)=0
$$

where $\nabla L G_{P}=\left[\frac{\partial L G_{P}}{\partial \omega}, \frac{\partial L G_{P}}{\partial R_{s}}, \frac{\partial L G_{P}}{\partial R_{c}}, \frac{\partial L G_{P}}{\partial E_{s y m}}, \frac{\partial L G_{P}}{\partial \mu_{1}}, \frac{\partial L G_{P}}{\partial \mu_{2}}\right]$. Further, $\mu_{k}^{*} \geq 0$ for $k=1,2$ if associated with an active inequality at $\Omega^{*}$, i.e.,

$$
\begin{cases}\mu_{1}^{*} \geq 0 & : \text { if } D_{s}+D_{v}=D_{0} \\ \mu_{1}^{*}=0 & : \text { otherwise }\end{cases}
$$

and

$$
\begin{cases}\mu_{2}^{*} \geq 0 & : \text { if } R_{s}+R_{c}=R_{0} \\ \mu_{2}^{*}=0 & : \text { otherwise }\end{cases}
$$

Constraint qualifications guarantee existence of unique Lagrange multipliers for a given local minimum $\Omega^{*}$ if the active inequality constraint gradients of (17) and (18) are linearly independent.

Considering the fact that the objective function (16) defined over a compact subset of $\mathcal{R}^{4}$ is continuously differentiable and the constraint gradients of (17) and (18) are linearly independent, finding the solution to the optimization problem is equivalent to finding the solutions of the equation set (22) specifying optimization variables $\omega, R_{s}, R_{c}$, and $E_{\text {sym }}$.

It is important to observe that the formulated problem of (16) is subject to discrete constraints applied to the source coding variable $\omega=\frac{1}{T}$ and the channel coding variable $r=\frac{k}{n}=$ $\frac{R_{s}}{R_{s}+R_{c}}$. Solving the problem of (16) is hence categorized under discrete constraint optimization problems which can be solved with the following approach. The approach is to add extra discrete constraints effectively changing the formulation of the optimization problem from a NonLinear Programming (NLP) to a Mixed Integer NonLinear Programming (MINLP) in which the variables $\omega$ and $R_{c}$ can only take on discrete values. In this approach, one selects the best solution among the set of solutions to the problems obtained for different discrete values of optimization parameters.

Taking the discrete constraints into consideration and assuming $p$ represents the number of parameter combinations, the time complexity of solving the problem of (22) with the above approach is $\mathcal{O}(p d \log d)$ where $d$ indicates the degree of (22). The complexity determines the overall complexity of the solution considering the fact that the rest of the calculations are in a lower time complexity order. The complexity result is quite good compared to a recursive optimization approach such as dynamic programming introducing a time complexity on the order of $\mathcal{O}\left(p d^{2}\right)$.

\section{NUMERICAL ANALYSIS}

In this section, we numerically validate our analytical results. Before proceeding with the explanation of our numerical results, we note that we are solving our problem for both single and double transmit antenna wireless systems. In the case of a double transmit antenna system, we assume that two signals are transmitted simultaneously from the two transmit antennas at each time slot. In addition, we assume that the slow fading wireless channel characterized by a Rayleigh distribution is quasi-static and flat implying that the path gains are constant over a frame but vary from one frame to another.

Considering the power limitation of the mobile nodes, our experiments cover uplink and mobile-to-mobile transmission scenarios. We compare the results of a single transmit antenna system with those of a double transmit antenna system. While our experiments span over source coding parameter settings associated with the sequences Containership.qcif, Foreman.qcif, MotherDaughter.qcif, News.qcif, and SilentVoice.qcif, we only report the results for Containership.qcif and Foreman.qcif. In addition, we select the scaling factors $\left[\epsilon_{s}, \epsilon_{c}, \epsilon_{t}\right]$ as $[1,0.01,1]$ indicating a transmission system with hardware encoding. Setting a block length of $n=222$ symbols for the RS coder with BPSK and QPSK modulations, we allow the source coding variable $T$ and channel coding variable $k$ to assume values from the discrete sets $[2,3,5,9,12,16,25,32]$ and $[40,50,60,70,80,90,100,112,122,132,142,152,162] \cup$ $[172,182,192,202]$, respectively. For a given bit rate $R_{0}$ of up to $256 \mathrm{Kbps}$ indicating the achievable bit rate of the $3 \mathrm{G}$ wireless standard, we plot the optimal power values obtained for maximum acceptable distortion measures. We map the distortion measure $D_{0}$ to $P S N R$ measure as $P S N R=10 \log _{10} \frac{255^{2}}{D_{0}}$. Utilizing BPSK modulation scheme, Fig. 2 and Fig. 3 plot the optimal values of $P_{\text {total }}$ versus different values of $P S N R$ for Containership.qcif and Foreman.qcif video sequences, respectively. Fig. 4 and Fig. 5 show similar curves utilizing QPSK modulation scheme. The results have been obtained for normalized values of $\mathcal{E}\left[|\alpha|^{2}\right]$ and $N_{0}$. The most important observation when comparing the figures is that the optimal power of a double antenna system is consistently lower than that of a single antenna system. Further, the following observations are in order. First, we observe that plotting the optimal values of power $P_{\text {total }}$ versus the values of available bit rate $R_{0}$ for a fixed quality of service $P S N R$ or $D_{0}$ yields similar qualitative results as the ones shown in the figures. However, it is observed that the impact of increasing the value of $R_{0}$ for a given $P S N R$ on the overall optimal power is not as significant as the impact of increasing the value of $P S N R$ for a given $R_{0}$. In other words, the two curves illustrated in different figures are closer to each other. Second, comparing the results of Containership.qcif with those of Foreman.qcif, we observe similar qualitative behaviors with higher optimal power values in the case of the second sequence. The results are expected considering the higher motion of the second sequence compared to the first. Finally, we note that the choice of scaling factors in our reported experiments indicates a scenario in which source and channel coders are implemented in hardware. The results of software implementation are similar and are not reported here. 


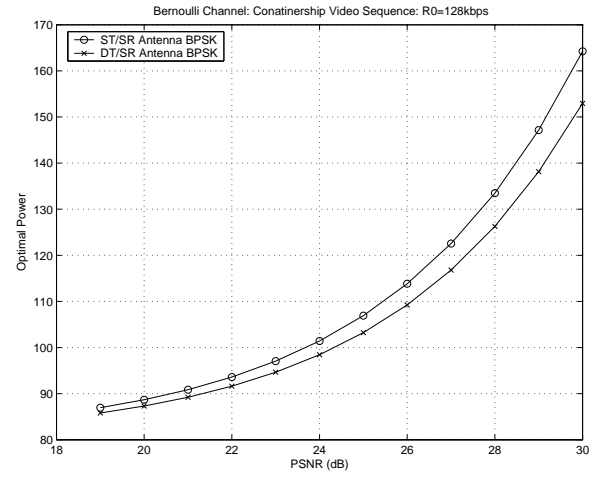

Fig. 2. BPSK plots of optimal power versus $P S N R$ for single and double transmit antenna systems. Containership video sequence has been utilized.

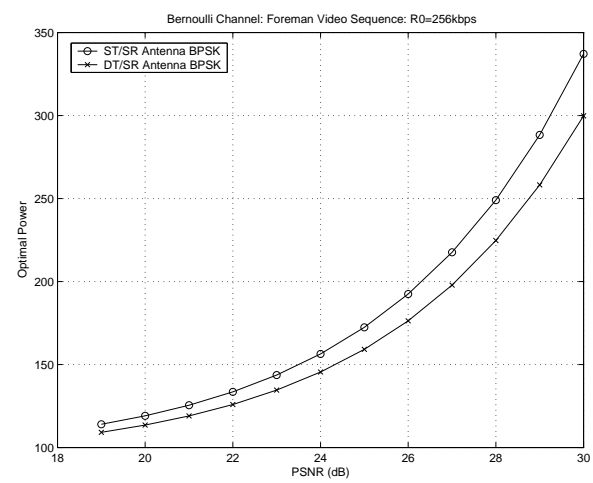

Fig. 3. BPSK plot of optimal power versus $P S N R$ for single and double transmit antenna systems. Foreman video sequence has been utilized.

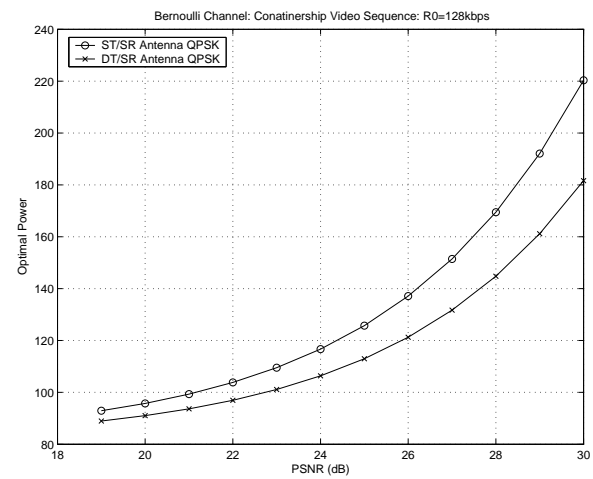

Fig. 4. QPSK plots of optimal power versus $P S N R$ for single and double transmit antenna systems. Containership video sequence has been utilized.

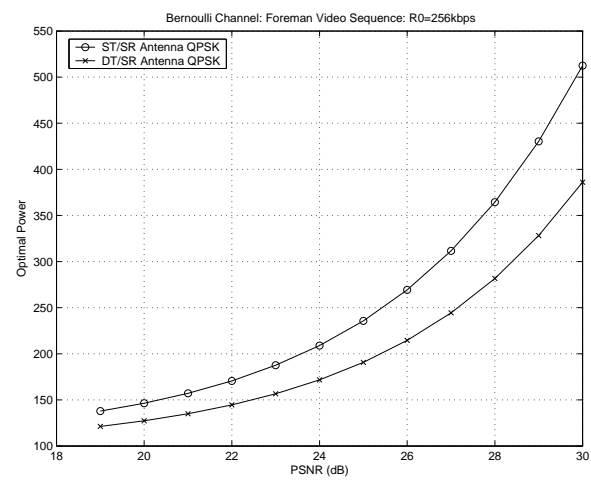

Fig. 5. QPSK plot of optimal power versus $P S N R$ for single and double transmit antenna systems. Foreman video sequence has been utilized.

\section{CONCLUSion}

In this paper, we presented an analytical solution to the problem of power control in wireless media systems with multiple transmit antennas. We provided an analysis of the underlying wireless system consisting of transmitting, channel, and receiving sides. Relying on our analysis results, we formulated an optimization problem aiming at minimizing the total power consumption of wireless media systems subject to a given quality of service level and an available bit rate. Our formulation considered the power consumption related to source coding, channel coding, and transmission of multiple transmit antennas respectively relying on the H.263 source coding standard, Rayleigh fading channels along with the Bernoulli loss model, and spacetime block codes. Finally, we evaluated the performance of our power optimized solution for both single and double transmit antenna systems. At the end, we would like to discuss some of the aspects of our future work. We are currently working on solving the same problem for wireless channels with memory relying on the Gilbert-Elliott loss model. Further, we are working on the expansion of our results into the layered and replicated media scenarios as a general combined framework for distributing multimedia traffic over the wireless backbone. We are focusing on both coding and networking aspects of the problem. In addition, we are developing novel content processing algorithms capable of providing video summaries, thereby further reducing the power consumption of a wireless system.

\section{REFERENCES}

[1] S. M. Alamouti, "A Simple Transmitter Diversity Scheme for Wireless Communications," IEEE JSAC, November 1998.

[2] S. Appadwedula, M. Goel, N. R. Shanbhad, D. L. Jones, K. Ramchandran, "Total System Energy Minimization for Wireless Image Transmission,” Journal of VLSI Signal Processing Systems, vol. 27, no. 1/2, pp. 99-117, February 2001.

[3] D. P. Bertsekas, "Nonlinear Programming, 2nd Edition," Athena Scientific Publishing, 1999.

[4] M. Goel, S. Appadwedula, N. R. Shanbhag, K. Ramchandran, and D.L. Jones, "A Low-Power Multimedia Communication System for Indoor Wireless Applications,” IEEE Workshop on SIPS99, 1999.

[5] P. J. M. Havinga, "Energy Efficiency of Error Correction on Wireless Systems," in Proc. of IEEE WCNC, Sept. 1999.

[6] Z. Ji, Q. Zhang, W. Zhu, Y-Q. Zhang, "End-to-End Power-Optimized Video Communication over Wireless Channels," in Proc. of IEEE Workshop on MMSP, 2001

[7] T. H. Lan and A. H. Tewfik, "Adaptive Low Power Multimedia Wireless Communications," in Proc. of IEEE Workshop on MMSP, 1997.

[8] X. Lu, Y. Wang, E. Erkip, "Power Efficient H.263 Video Transmission over Wireless Channels," in Proc. of IEEE ICIP, 2002.

[9] M. K. Simon, M. S. Alouini, "Digital Communication over Fading Channels: A Unified Approach to Performance Analysis," John Wiley, ISBN: 0471317799, 2000.

[10] M. K. Simon, "Evaluation of Average Bit Error Probability for SpaceTime Coding Based on a Simpler Exact Evaluation of Pairwise Error Probability," Int'l Journal on Communications and Networks, September 2001.

[11] K. Stuhlmuller, N. Farber, M. Link, B. Girod, "Analysis of Video Transmission over Lossy Channels," IEEE JSAC, June 2000.

[12] V. Tarokh, H. Jafarkhani, A. R. Calderbank, "Space-Time Block Coding from Orthogonal Designs,” IEEE Trans. Inform. Theory, July 1999. 